BULL. AUSTRAL. MATH. SOC.

$1.6 A 08,16 A 46,18 E 40$

VOL. II (1974), 425-428.

\title{
A characterization of left semiartinian rings
}

\section{Jonathan S. Golan}

In defining the torsion-theoretic Krull dimension of an associative ring $R$ we make use of a function $\delta$ from the complete lattice of all subsets of the torsion-theoretic spectrum of $R$ to the complete lattice of all hereditary torsion theories on $R$-mod . In this note we give necessary and sufficient conditions for $\delta$ to be injective, surjective, and bijective. In particular, $\delta$ is bijective if and only if $R$ is a left semiartinian ring.

Throughout the following $R$ will always designate an associative (but not necessarily commutative) ring with unit element and $R$-tors will denote the complete lattice of all hereditary torsion theories on the category $R$-mod of unitary left $R$-modules. If $\tau \in R$-tors, we denote by $\tau_{\tau}$ the class of all $\tau$-torsion left $R$-modules, by $F_{\tau}$ the class of all $\tau$-torsionfree left $R$-modules, and by $T_{\tau}(-)$ the $\tau$-torsion radical. The smallest element $\xi$ of $R$-tors is characterized by $T_{\xi}=\{0\}$; the largest element $X$ of $R$-tors is characterized by $F_{X}=\{0\}$. If $M$ is a left $R$-module, we denote by $\chi(M)$ the largest element of $R$-tors relative to which $M$ is torsionfree.

If $\tau \in R$-tors, a nonzero left $R$-module $M$ is said to be $\tau$-cocritical if and only if $M$ is $\tau$-torsionfree while $M / N$ is $\tau$-torsion for every nonzero submodule $N$ of $M$. A nonzero left $R$-module $M$ is said to be cocritical if and only if it is $X(M)$-cocritical. The elements Received 23 August 1974. 
of $R$-tors of the form $\pi=\chi(M)$ for $M$ a cocritical left $R$-module are called prime torsion theories [2]. The set of all prime elements of $R$-tors is called the left spectmom of $R$ and is denoted by $R$-sp . If $M$ is a left $R$-module, we define the assassin of $M$ by

$$
\operatorname{ass}(M)=\{\pi \in R \text {-sp } \mid M \text { has a } \pi \text {-cocritical submodule }\} \text {. }
$$

A ring $R$ is said to be left semiartinian if and only if every nonzero left $R$-module has a simple submodule.

In [1] we defined the function

$$
\delta: \text { subsets of } R \text {-sp } \rightarrow R \text {-tors }
$$

as follows: if $U \subseteq R$-sp then

$$
T_{\delta(U)}=\left\{R^{M} \mid \varnothing \neq \text { ass }(M / N) \subseteq U \text { for every proper submodule } N \text { of } M\right\} .
$$

This function is used in defining a dimension for rings analogous to the classical Krull dimension for commutative rings. In this note we wish to point out some properties of the function $\delta$ itself. It is easy and straightforward to check that $\delta$ is a morphism of complete lattices and that $\delta(\emptyset)=\xi$.

PROPOSITION 1. The following conditions are equivalent:

(1) $\delta$ is injective;

(2) if $\pi \in R$-sp then there exists a $\pi$-cocritical simple left $R$-moduze.

Proof. (I) $\Rightarrow(2)$. If $\pi \in R$-sp then $\delta(\{\pi\}) \neq \delta(\emptyset)=\xi$ by the injectiveness of $\delta$ and so there exists a nonzero left $R$-module $M$ which is $\delta(\{\pi\})$-torsion. In particular $M$ has a $\pi$-cocritical submodule $M^{\prime}$. If $0 \neq N$ is a proper submodule of $M^{\prime}$ then ass $\left(M^{\prime} / N\right)=\{\pi\}$ and so $M^{\prime} / N$ has a $\pi$-cocritical submodule. But $M^{\prime} / N$ is $\pi$-torsion and so we have a contradiction. Thus $M^{\prime}$ can have no proper submodules other than 0 and so $M^{\prime}$ is simple.

(2) $\Rightarrow$ (1). Assume that $\delta(U)=\delta\left(U^{\prime}\right)$ for $U \neq U^{\prime} \subseteq R$-sp. Without loss of generality we can assume that there exists a $\pi \in U \backslash U^{\prime}$. If $M$ is a simple $\pi$-cocritical left $R$-module then $M \in T_{\delta(U)}=T_{\delta\left(U^{\prime}\right)}$ and so $\pi \in U^{\prime}-$ a contradiction. Thus $\delta$ is injective.

PROPOSITION 2. The following conditions are equivalent: 
(1) $\delta$ is surjective;

(2) $\emptyset \neq \operatorname{ass}(M)$ for every nonzero left R-module $M$.

Proof. (1) $\Rightarrow(2)$. If $\delta$ is surjective then there exists a $U \subseteq R$-sp for which $\delta(U)=\mathrm{X}$. Therefore $T_{\delta(U)}=R$-mod which implies (2).

(2) $\Rightarrow(1)$. Let $\tau \in R$-tors and let $U=U\left\{\operatorname{ass}(M) \mid 0 \neq M \in T_{\tau}\right\}$. Then $\emptyset \neq \operatorname{ass}(M / N) \subseteq U$ for every proper submodule $N$ of $M \in T_{\tau}$ and so $\tau \leq \delta(U)$. Assume that $\tau \neq \delta(U)$ and let $0 \neq M \in T_{\delta(U)} \backslash T_{\tau}$. Then we have $0 \neq \bar{M}=M / T_{\tau}(M) \in T_{\delta(U)} \cap F_{\tau}$. Let $\pi \in \operatorname{ass}(\bar{M})$ and let $N$ be a $\pi$-cocritical submodule of $\bar{M}$. Then $\pi=\chi(N) \geq \tau$. But $\bar{M} \in T_{\delta(U)}$ and so $\pi \in U$. Therefore there exists a $\pi$-cocritical left $R$-module $N^{\prime} \in T_{\tau} \subseteq T_{\pi}$, a contradiction. Therefore we must have $\tau=\delta(U)$.

PROPOSITION 3. The following conditions are equivalent:

(1) $\delta$ is bijective;

(2) $R$ is a left semiartinian ring.

Proof. (1) $\Rightarrow(2)$. Let $M$ be a nonzero left $R$-module. By Proposition 2, $\varnothing \neq$ ass $(M)$. If $\pi \in \operatorname{ass}(M)$ then by Proposition 1 there exists a simple left $R$-module $N^{\prime}$ which is $\pi$-cocritical. Moreover, $M$ has a $\pi$-cocritical submodule $N$. Since $N^{\prime}$ is $\pi$-torsionfree, $\operatorname{hom}_{R}\left(N^{\prime}, E(N)\right) \neq 0$. Since $N^{\prime}$ is $\pi$-cocritical and $E(N)$ is $\pi$-torsionfree, any nonzero homomorphism $\alpha: N^{\prime} \rightarrow E(N)$ is a monomorphism. Since $N^{\prime}$ is simple, $N^{\prime} \alpha \subseteq N$. Therefore $M$ has a simple submodule. This proves that $R$ is left semiartinian.

(2) $\Rightarrow$ (1). If $M$ is a nonzero left $R$-module then by (2), $M$ has a simple submodule. Since all simple left $R$-modules are cocritical, this implies that ass $(M) \neq \emptyset$. If $\pi \in R$-sp and $N$ is a $\pi$-cocritical left $R$-module then $N$ has a simple submodule $N^{\prime}$ which is also $\pi$-cocritical and so $\pi=X\left(N^{\prime}\right)$. By Propositions 1 and $2, \delta$ is then bijective.

PROPOSITION 4. If $R$ is a left semiartinian ring then $\delta^{-1}$ is defined by $\delta^{-1}: \tau \mapsto\{\chi(M) \mid M$ is simple and $\tau$-torsion $\}$.

Proof. Let $\tau \in R$-tors and let 


$$
U=\{\chi(M) \mid M \text { is simple and } \tau \text {-torsion }\} \text {. }
$$

If $M$ is a $\tau$-torsion left $R$-module then so is $M / N$ for every proper submodule $N$ of $M$. If $\pi \in$ ass $(M / N)$ then there exists a $\pi$-cocritical submodule $M^{\prime} / N$ of $M / N$. Since $R$ is left semiartinian, $M^{\prime} / N$ in turn has a simple submodule $M^{\prime \prime} / N$ which is also $\tau$-torsion and $\tau$-cocritical. Then $\pi=\chi\left(M^{\prime \prime} / N\right) \in U$. Hence $T_{\tau} \subseteq T_{\delta(U)}$.

Conversely assume that $M$ is a left $R$-module which is not T-torsion. Then $0 \neq \bar{M}=M / T_{\tau}(M)$ and so $\bar{M}$ has a simple submodule $N^{\prime}$ which is $\tau$-torsionfree. This implies that $\chi\left(N^{\prime}\right) \in$ ass $(\bar{M}) \backslash U$ and so $M \notin T_{\delta(U)}$.

\section{References}

[1] Jonathan S. Golan, "A Krull-like dimension for noncommutative rings", Israel J. Math. (to appear).

[2] Oscar Goldman, "Rings and modules of quotients", J. AZgebra 13 (1969), $10-47$.

Department of Mathematics,

University of Haifa,

Mt Carmel,

Haifa,

Israel. 